25 Ikebe H, Terubayashi H, Akagi Y, Kodor PF, Kinoshita JM. Ciliary body changes associate with aldose reductase in galactosemic rats. Acta Soc Ophthalmol 1989; 93: 758-62.

26 Kato S, Oshika T, Funatsu H, Kokutei K, Yamashita H, Sawa $M$. Aqueous protein concentration in diabetics. Report 5. Relationship between aqueous protein concentration and pathological findings of iridal vessels. Acta Soc Ophthalmol f pn 1992; 96: 1000-6.

27 Kato S, Oshika T, Funatsu H, Katsuro K, Yamashita H, Sawa $M$. Aqueous protein concentration in diabetics 4 . Implication of retinal 1992; 46: 149-53.

28 Schonherr U, Kuchle $M$, Nguyen NX, Steinhauser B, Naumann GOH. Capillary exudation in diabetic retinopathy and diabetic iridopathy-correlation between fluorescein angiography and laser flare cell meter. Inves Ophthalmol Vis Sci (Suppl) 1992; 33: 1364
29 Azumi A, Inoue $M$, Yamamoto $M$. Application of laser flarecell meter in patients with diabetes mellitus 2, Factors influencing the flare counts. Folia Ophthalmol fpn 1990; 41: influencing $986-90$.

30 Hayashi M, Yablonski ME, Boxrud C, Fong N, Berger C, Javanovic LJ. Decreased formation of aqueous humor in insulin-dependent diabetic patients. Br F Ophthalmol 1989 73: 621-3.

31 Ogawa T, Ohara K, Shimizu H. Correlation between total aqueous protein concentration and photon counts in rabbits. Acta Soc Ophthalmol.7pn 1990; 94: 1001-6.

32 Shah SM, Spalton DJ, Taylor JC. Correlations between laser flare measurements and anterior chamber protein concentrations. Invest Ophthalmol Vis Sci 1992; 33: 2878-84.

33 Dernouchamps JP. The protein of the aqueous humor. Doc Ophthalmol 1982; 53: 193-248.

\title{
History of ophthalmology
}

\section{Antisepsis in ophthalmology}

Antisepsis came to surgery, and to ophthalmology, in the $1880 \mathrm{~s}$, and like all innovations it was much debated. Its true origins were probably in Vienna, where the obstetric ward staffed by midwives had a reasonable mortality for the time. However, the adjacent ward staffed by medical students, who came directly from the dissecting room, had such a reputation that women assigned to it literally wept and begged to be allowed to die at home. The midwives probably explained this by blaming the gross incompetence of the medical students, until a certain Dr Semmelweiss began to look further. Eliminating differences in patients' social class, food, window opening, degree of noise from the street, etc between the two wards, he decided that students were bringing in some contagion from their dissections, and by introducing them forcibly to bowls of lime chloride he dramatically reduced the mortality.

It was Joseph Lister who, several years later, closed his books after an extensive study of Semmelweiss and Pasteur and decided that airborne organisms must be eliminated by chemical antisepsis. Having seen carbolic acid used as a sewerage disinfectant in Carlisle, he felt it should be adequate for the task and published his findings extensively throughout the 1870s. As various colleagues replicated his results, they were generally accepted.

Von Graefe suggested that antisepsis be used in cataract extraction, and Simeon Snell, returning from holiday to read his account, decided to give the method a trial. For his next dozen patients, eyes were washed preoperatively in $2 \%$ carbolic acid (presumably borrowed from his housekeeper for the occasion) as were instruments and sponges. Boracic acid was applied to the dressings afterwards. (Charles Darwin, momentarily diverted from evolution, suggested the latter substance to Lister.) Snell reported a satisfying lack of suppuration, yet a distressing amount of patient complaint as the solution was applied. Furthermore, the degree of conjunctival swelling made the elevation of the lid technically difficult. Snell, combining theory with practicality, returned the carbolic acid to his cleaner and vowed to use thymol solution in a future series.
By the end of the 1890s, knowledge had advanced, and the prestigious Bowman lecture on ophthalmology summarised the new developments - namely, that normal tissue possessed its own defences, and thus that extremely strong chemical agents should not be used. Furthermore, from experimental insertion of gold and quicksilver into rabbit eyes, it was seen that inflammation could be caused both by infection and also by some (but not all) foreign bodies.

By 1898 , heat sterilisation had arrived and largely replaced chemical antisepsis in eye operations. McGillivray describes his regimen for aseptic cataract extraction in detail. Firstly, the patient had a bath and change of underclothes (and this in itself could be quite a shock to the poorer subject), then a test dressing was applied overnight which must remain unstained for the operation to proceed. The surgeon washed his hands in water, then weak antiseptic, then sterile saline, and superintended the boiling of his instruments for no more than 2 minutes (any longer blunted the scalpel blades, and the cries from hospital surgeons for more than one set of instruments each were piteous). The eye was then laboriously cleaned with a sterile mop, irrigated with streams of saline at $90^{\circ} \mathrm{F}$, flushed out with further saline poured from a height, and the area beneath the lids was attacked with a saline douche.

McGillivray found that saline had a 'soothing' effect when applied to the eye, and in contrast with the howls accompanying direct application of carbolic acid, this was undoubtedly true.

The subject of hand disinfection was investigated by incubating scrapings from beneath the operator's nails. One surgeon found that his own hands, and the nurses' could be sterilised by a moderate sublimate solution. The finding that the house officer was invariably 'contaminated' would have been no surprise to Semmelweiss, but probably led to the prescription of a much more caustic disinfectant for that unfortunate individual.

F ROMAN

Leber MD. Present position of our knowledge of inflammation. Trans Ophthalmol Soc UK 1892; 13: 1-14.

McGillivray A. The aseptic treatment of wounds in cataract surgery. Trans Ophthalmol Soc UK 1898; 18: 320-42. Snell S. Antisepsis in cataract extraction. Br Med $\mathcal{F} 1880$; i: 241. 